\title{
Erratum: Hunting for vectorlike quarks
}

\author{
Radovan Dermíšek, ${ }^{a}$ Enrico Lunghi ${ }^{a}$ and Seodong Shin ${ }^{b, c}$ \\ ${ }^{a}$ Physics Department, Indiana University, \\ 727 E. Third St., Bloomington, IN 47405, U.S.A. \\ ${ }^{b}$ Enrico Fermi Institute, University of Chicago, \\ 933 East 56th Street, Chicago, IL 60637, U.S.A. \\ ${ }^{c}$ Department of Physics and IPAP, Yonsei University, \\ 50 Yonsei-ro, Seoul 03722, Korea \\ E-mail: dermisek@indiana.edu, elunghi@indiana.edu, \\ shinseod@indiana.edu
}

ERrATUM TO: JHEP04(2019)019

ArXIV EPRINT: 1901.03709 
We found a mistake (involving a sign and factor of $\sqrt{2}$ ) which propagates through tables $2-5$ and several equations presented in the appendix. The two lower panels of figure 1 are mildly modified while all other numerical results are unaffected. In tables $3-5$ we adopt a more useful parameterization for the branching ratios in the case of a vectorlike quark doublet with couplings to $H_{u}$ and $H_{d}$.

\section{Corrected versions of tables $2-5$.}

\begin{tabular}{|c|c|c|}
\hline$\frac{64 \pi}{m_{t_{4}}} \Gamma$ & doublet & singlet \\
\hline \hline$t_{4} \rightarrow W^{+} b$ & $2 \lambda_{Q}^{2} c_{\beta}^{2}$ & $2 \kappa_{T}^{2} s_{\beta}^{2}$ \\
\hline$t_{4} \rightarrow Z t$ & $\kappa_{Q}^{2} s_{\beta}^{2}$ & $\kappa_{T}^{2} s_{\beta}^{2}$ \\
\hline$t_{4} \rightarrow h t$ & $\kappa_{Q}^{2} s_{\beta}^{2}$ & $\kappa_{T}^{2} s_{\beta}^{2}$ \\
\hline$t_{4} \rightarrow H t$ & $\kappa_{Q}^{2} c_{\beta}^{2}$ & $\kappa_{T}^{2} c_{\beta}^{2}$ \\
\hline$t_{4} \rightarrow H^{+} b$ & $2 \lambda_{Q}^{2} s_{\beta}^{2}$ & $2 \kappa_{T}^{2} c_{\beta}^{2}$ \\
\hline
\end{tabular}

\begin{tabular}{|c|c|c|}
\hline$\frac{64 \pi}{m_{b_{4}}} \Gamma$ & doublet & singlet \\
\hline \hline$b_{4} \rightarrow W^{+} t$ & $2 \kappa_{Q}^{2} s_{\beta}^{2}$ & $2 \lambda_{B}^{2} c_{\beta}^{2}$ \\
\hline$b_{4} \rightarrow Z b$ & $\lambda_{Q}^{2} c_{\beta}^{2}$ & $\lambda_{B}^{2} c_{\beta}^{2}$ \\
\hline$b_{4} \rightarrow h b$ & $\lambda_{Q}^{2} c_{\beta}^{2}$ & $\lambda_{B}^{2} c_{\beta}^{2}$ \\
\hline$b_{4} \rightarrow H b$ & $\lambda_{Q}^{2} s_{\beta}^{2}$ & $\lambda_{B}^{2} s_{\beta}^{2}$ \\
\hline$b_{4} \rightarrow H^{+} t$ & $2 \kappa_{Q}^{2} c_{\beta}^{2}$ & $2 \lambda_{B}^{2} s_{\beta}^{2}$ \\
\hline
\end{tabular}

Table 2. Leading dependence of the $t_{4}$ and $b_{4}$ decay widths on the Lagrangian parameters in the large mass limit. We use the shorthand notation $s_{\beta}=\sin \beta$ and $c_{\beta}=\cos \beta$.

\begin{tabular}{|c|c|c|c|c|c|c|}
\hline & \multicolumn{2}{|c|}{ Couplings to $H_{u}$ only } & \multicolumn{2}{c|}{ Couplings to $H_{d}$ only } & \multicolumn{2}{c|}{ Couplings to $H_{u}$ and $H_{d}$} \\
\cline { 2 - 7 } & doublet & singlet & doublet & singlet & doublet & singlet \\
\hline \hline $\mathrm{BR}\left(t_{4} \rightarrow W^{+} b\right)$ & 0 & $1 / 2$ & 1 & 0 & $(1-x) \frac{1}{1+x\left(t_{\beta}^{2}-1\right)}$ & $1 / 2$ \\
\hline $\mathrm{BR}\left(t_{4} \rightarrow Z t\right)$ & $1 / 2$ & $1 / 4$ & 0 & 0 & $\frac{x}{2} \frac{t_{\beta}^{2}}{1+x\left(t_{\beta}^{2}-1\right)}$ & $1 / 4$ \\
\hline $\mathrm{BR}\left(t_{4} \rightarrow h t\right)$ & $1 / 2$ & $1 / 4$ & 0 & 0 & $\frac{x}{2} \frac{t_{\beta}^{2}}{1+x\left(t_{\beta}^{2}-1\right)}$ & $1 / 4$ \\
\hline \hline $\operatorname{BR}\left(b_{4} \rightarrow W^{+} t\right)$ & 1 & 0 & 0 & $1 / 2$ & $\frac{t_{\beta}^{2}}{1+x\left(t_{\beta}^{2}-1\right)}$ & $1 / 2$ \\
\hline $\operatorname{BR}\left(b_{4} \rightarrow Z b\right)$ & 0 & 0 & $1 / 2$ & $1 / 4$ & $\frac{1-x}{2} \frac{1}{1+x\left(t_{\beta}^{2}-1\right)}$ & $1 / 4$ \\
\hline $\operatorname{BR}\left(b_{4} \rightarrow h b\right)$ & 0 & 0 & $1 / 2$ & $1 / 4$ & $\frac{1-x}{2} \frac{1}{1+x\left(t_{\beta}^{2}-1\right)}$ & $1 / 4$ \\
\hline
\end{tabular}

Table 3. Vectorlike quark branching ratios in the Goldstone boson equivalence limit. When couplings to both $H_{u}$ and $H_{d}$ are present the branching ratios are functions of $t_{\beta}=\tan \beta$ and $x=\kappa_{Q}^{2} /\left(\kappa_{Q}^{2}+\lambda_{Q}^{2}\right)$. 


\begin{tabular}{|c|c|c|c|c|c|c|}
\hline & \multicolumn{2}{|c|}{ Couplings to $H_{u}$ only } & \multicolumn{2}{c|}{ Couplings to $H_{d}$ only } & \multicolumn{2}{c|}{ Couplings to $H_{u}$ and $H_{d}$} \\
\cline { 2 - 7 } & doublet & singlet & doublet & singlet & doublet & singlet \\
\hline \hline $\mathrm{BR}\left(t_{4} \rightarrow W^{+} b\right)$ & 0 & $\frac{2 t_{\beta}^{2}}{4 t_{\beta}^{2}+1}$ & 1 & 0 & $\frac{2(1-x)}{2+x\left(2 t_{\beta}^{2}-1\right)}$ & $\frac{2 t_{\beta}^{2}}{4 t_{\beta}^{2}+1}$ \\
\hline $\mathrm{BR}\left(t_{4} \rightarrow Z t\right)$ & $\frac{t_{\beta}^{2}}{2 t_{\beta}^{2}+1}$ & $\frac{t_{\beta}^{2}}{4 t_{\beta}^{2}+1}$ & 0 & 0 & $\frac{x t_{\beta}^{2}}{2+x\left(2 t_{\beta}^{2}-1\right)}$ & $\frac{t_{\beta}^{2}}{4 t_{\beta}^{2}+1}$ \\
\hline $\mathrm{BR}\left(t_{4} \rightarrow h t\right)$ & $\frac{t_{\beta}^{2}}{2 t_{\beta}^{2}+1}$ & $\frac{t_{\beta}^{2}}{4 t_{\beta}^{2}+1}$ & 0 & 0 & $\frac{x t_{\beta}^{2}}{2+x\left(2 t_{\beta}^{2}-1\right)}$ & $\frac{t_{\beta}^{2}}{4 t_{\beta}^{2}+1}$ \\
\hline $\mathrm{BR}\left(t_{4} \rightarrow H t\right)$ & $\frac{1}{2 t_{\beta}^{2}+1}$ & $\frac{1}{4 t_{\beta}^{2}+1}$ & 0 & 0 & $\frac{x}{2+x\left(2 t_{\beta}^{2}-1\right)}$ & $\frac{1}{4 t_{\beta}^{2}+1}$ \\
\hline \hline $\operatorname{BR}\left(b_{4} \rightarrow W+t\right)$ & 1 & 0 & 0 & $\frac{2}{t_{\beta}^{2}+4}$ & $\frac{2 x t_{\beta}^{2}}{t_{\beta}^{2}+2+x\left(t_{\beta}^{2}-2\right)}$ & $\frac{2}{t_{\beta}^{2}+4}$ \\
\hline $\mathrm{BR}\left(b_{4} \rightarrow Z b\right)$ & 0 & 0 & $\frac{1}{t_{\beta}^{2}+2}$ & $\frac{1}{t_{\beta}^{2}+4}$ & $\frac{1-x}{t_{\beta}^{2}+2+x\left(t_{\beta}^{2}-2\right)}$ & $\frac{1}{t_{\beta}^{2}+4}$ \\
\hline $\operatorname{BR}\left(b_{4} \rightarrow h b\right)$ & 0 & 0 & $\frac{1}{t_{\beta}^{2}+2}$ & $\frac{1}{t_{\beta}^{2}+4}$ & $\frac{1-x}{t_{\beta}^{2}+2+x\left(t_{\beta}^{2}-2\right)}$ & $\frac{1}{t_{\beta}^{2}+4}$ \\
\hline $\operatorname{BR}\left(b_{4} \rightarrow H b\right)$ & 0 & 0 & $\frac{t_{\beta}^{2}}{t_{\beta}^{2}+2}$ & $\frac{t_{\beta}^{2}}{t_{\beta}^{2}+4}$ & $\frac{(1-x) t_{\beta}^{2}}{t_{\beta}^{2}+2+x\left(t_{\beta}^{2}-2\right)}$ & $\frac{t_{\beta}^{2}}{t_{\beta}^{2}+4}$ \\
\hline
\end{tabular}

Table 4. Branching ratios of the vectorlike quarks $t_{4}$ and $b_{4}$ into $W, Z, h$ and $H$ as a function of $t_{\beta}=\tan \beta$ and $x=\kappa_{Q}^{2} /\left(\kappa_{Q}^{2}+\lambda_{Q}^{2}\right)$. These expressions are an excellent approximation for heavy vectorlike masses and in the limit in which the couplings in table 2 dominate.

\begin{tabular}{|c|c|c|c|c|c|c|}
\hline & \multicolumn{2}{|c|}{ Couplings to $H_{u}$ only } & \multicolumn{2}{c|}{ Couplings to $H_{d}$ only } & \multicolumn{2}{c|}{ Couplings to $H_{u}$ and $H_{d}$} \\
\cline { 2 - 7 } & doublet & singlet & doublet & singlet & doublet & singlet \\
\hline \hline $\mathrm{BR}\left(t_{4} \rightarrow W^{+} b\right)$ & 0 & $\frac{2 t_{\beta}^{2}}{4 t_{\beta}^{2}+2}$ & $\frac{1}{t_{\beta}^{2}+1}$ & 0 & $\frac{1-x}{1-x+t_{\beta}^{2}}$ & $\frac{2 t_{\beta}^{2}}{4 t_{\beta}^{2}+2}$ \\
\hline $\mathrm{BR}\left(t_{4} \rightarrow Z t\right)$ & $1 / 2$ & $\frac{t_{\beta}^{2}}{4 t_{\beta}^{2}+2}$ & 0 & 0 & $\frac{x}{2} \frac{t_{\beta}^{2}}{1-x+t_{\beta}^{2}}$ & $\frac{t_{\beta}^{2}}{4 t_{\beta}^{2}+2}$ \\
\hline $\mathrm{BR}\left(t_{4} \rightarrow h t\right)$ & $1 / 2$ & $\frac{t_{\beta}^{2}}{4 t_{\beta}^{2}+2}$ & 0 & 0 & $\frac{x}{2} \frac{t_{\beta}^{2}}{1-x+t_{\beta}^{2}}$ & $\frac{t_{\beta}^{2}}{4 t_{\beta}^{2}+2}$ \\
\hline $\mathrm{BR}\left(t_{4} \rightarrow H^{+} b\right)$ & 0 & $\frac{2}{4 t_{\beta}^{2}+2}$ & $\frac{t_{\beta}^{2}}{t_{\beta}^{2}+1}$ & 0 & $\frac{(1-x) t_{\beta}^{2}}{1-x+t_{\beta}^{2}}$ & $\frac{2}{4 t_{\beta}^{2}+2}$ \\
\hline \hline $\operatorname{BR}\left(b_{4} \rightarrow W^{+} t\right)$ & $\frac{t_{\beta}^{2}}{t_{\beta}^{2}+1}$ & 0 & 0 & $\frac{2}{2 t_{\beta}^{2}+4}$ & $\frac{x t_{\beta}^{2}}{1+x t_{\beta}^{2}}$ & $\frac{2}{2 t_{\beta}^{2}+4}$ \\
\hline $\operatorname{BR}\left(b_{4} \rightarrow Z b\right)$ & 0 & 0 & $1 / 2$ & $\frac{1}{2 t_{\beta}^{2}+4}$ & $\frac{1-x}{2} \frac{1}{1+x t_{\beta}^{2}}$ & $\frac{1}{2 t_{\beta}^{2}+4}$ \\
\hline $\operatorname{BR}\left(b_{4} \rightarrow h b\right)$ & 0 & 0 & $1 / 2$ & $\frac{1}{2 t_{\beta}^{2}+4}$ & $\frac{1-x}{2} \frac{1}{1+x t_{\beta}^{2}}$ & $\frac{1}{2 t_{\beta}^{2}+4}$ \\
\hline $\operatorname{BR}\left(b_{4} \rightarrow H^{+} t\right)$ & $\frac{1}{t_{\beta}^{2}+1}$ & 0 & 0 & $\frac{2 t_{\beta}^{2}}{2 t_{\beta}^{2}+4}$ & $\frac{x}{1+x t_{\beta}^{2}}$ & $\frac{2 t_{\beta}^{2}}{2 t_{\beta}^{2}+4}$ \\
\hline
\end{tabular}

Table 5. Branching ratios of the vectorlike quarks $t_{4}$ and $b_{4}$ into $W, Z, h$ and $H^{ \pm}$as a function of $t_{\beta}=\tan \beta$ and $x=\kappa_{Q}^{2} /\left(\kappa_{Q}^{2}+\lambda_{Q}^{2}\right)$. These expressions are an excellent approximation for heavy vectorlike masses and in the limit in which the couplings in table 2 dominate. 
Bottom two panels of figure 1.
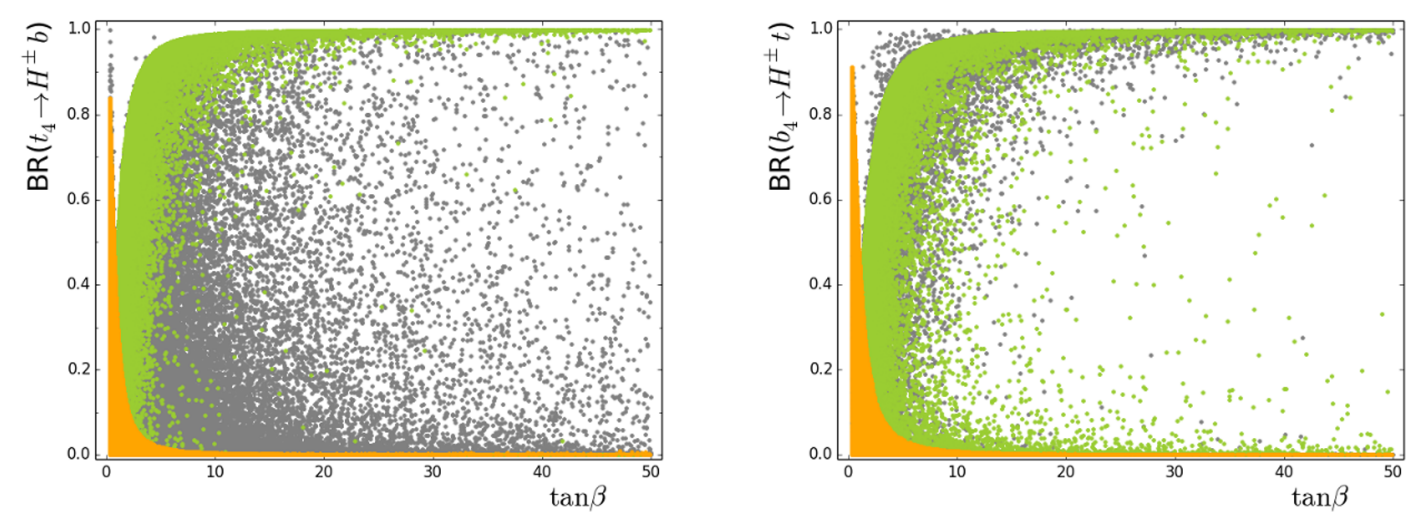

\section{Correct version of several equations.}

$$
\begin{aligned}
& \left(\begin{array}{c}
G \\
A
\end{array}\right)=\left(\begin{array}{cc}
\cos \beta & \sin \beta \\
-\sin \beta & \cos \beta
\end{array}\right)\left(\begin{array}{c}
\sqrt{2}\left(\operatorname{Im} H_{d}^{0}\right) \\
\sqrt{2}\left(-\operatorname{Im} H_{u}^{0}\right)
\end{array}\right) \\
& \mathcal{L}_{A}=-\frac{i}{\sqrt{2}} \overline{\hat{u}}_{L} V_{L}^{u \dagger} Y_{u}^{A} V_{R}^{u} \hat{u}_{R}(-A \cos \beta) \\
& -\frac{i}{\sqrt{2}} \overline{\hat{d}}_{L} V_{L}^{d \dagger} Y_{d}^{A} V_{R}^{d} \hat{d}_{R}(-A \sin \beta)+\text { h.c. }, \\
& \lambda_{u_{a} u_{b}}^{A}=-i \cos \beta\left(V_{L}^{u \dagger} Y_{u}^{A} V_{R}^{u}\right)_{a b}, \\
& \left(\begin{array}{c}
G^{ \pm} \\
H^{ \pm}
\end{array}\right)=\left(\begin{array}{cc}
\cos \beta & \sin \beta \\
-\sin \beta & \cos \beta
\end{array}\right)\left(\begin{array}{c}
H_{d}^{ \pm} \\
-H_{u}^{ \pm}
\end{array}\right) \\
& \mathcal{L}_{H^{ \pm}}=-\overline{\hat{u}}_{L} V_{L}^{u \dagger} Y_{d}^{H^{ \pm}} V_{R}^{d} \hat{d}_{R} H^{+} \\
& -\overline{\hat{d}}_{L} V_{L}^{d \dagger} Y_{u}^{H^{ \pm}} V_{R}^{u} \hat{u}_{R} H^{-}+\text {h.c. }, \\
& Y_{u}^{H^{ \pm}}=-\cos \beta\left(\begin{array}{ccc}
y_{t} & 0 & \kappa_{T} \\
\kappa_{Q} & 0 & \kappa \\
0 & \bar{\lambda} \tan \beta & 0
\end{array}\right) \quad \text { and } \quad Y_{d}^{H^{ \pm}}=-\sin \beta\left(\begin{array}{ccc}
y_{b} & 0 & \lambda_{B} \\
\lambda_{Q} & 0 & \lambda \\
0 & \bar{\kappa} / \tan \beta & 0
\end{array}\right) . \\
& \mathcal{L}_{H^{ \pm}}=-\overline{\hat{u}}_{L a} \lambda_{u_{a} d_{b}}^{H^{ \pm}} \hat{d}_{R b} H^{+}-\overline{\hat{d}}_{L a} \lambda_{d_{a} u_{b}}^{H^{ \pm}} \hat{u}_{R b} H^{-}+\text {h.c. },
\end{aligned}
$$




$$
\begin{aligned}
& \lambda_{t_{4} b}^{H^{ \pm}}=-\sin \beta \cdot\left[y_{b}\left(V_{L}^{u}\right)_{43}^{\dagger}\left(V_{R}^{d}\right)_{33}+\lambda_{B}\left(V_{L}^{u}\right)_{43}^{\dagger}\left(V_{R}^{d}\right)_{53}+\lambda_{Q}\left(V_{L}^{u}\right)_{44}^{\dagger}\left(V_{R}^{d}\right)_{33}+\lambda\left(V_{L}^{u}\right)_{44}^{\dagger}\left(V_{R}^{d}\right)_{53}\right] \\
& -\cos \beta \bar{\kappa}\left(V_{L}^{u}\right)_{45}^{\dagger}\left(V_{R}^{d}\right)_{43}, \\
& \lambda_{b t_{4}}^{H^{ \pm}}=-\cos \beta \cdot\left[y_{t}\left(V_{L}^{d}\right)_{33}^{\dagger}\left(V_{R}^{u}\right)_{34}+\kappa_{T}\left(V_{L}^{d}\right)_{33}^{\dagger}\left(V_{R}^{u}\right)_{54}+\kappa_{Q}\left(V_{L}^{d}\right)_{34}^{\dagger}\left(V_{R}^{u}\right)_{34}+\kappa\left(V_{L}^{d}\right)_{34}^{\dagger}\left(V_{R}^{u}\right)_{54}\right] \\
& -\sin \beta \cdot \bar{\lambda}\left(V_{L}^{d}\right)_{35}^{\dagger}\left(V_{R}^{u}\right)_{44} \text {, } \\
& \lambda_{b_{4} t}^{H^{ \pm}}=-\cos \beta \cdot\left[y_{t}\left(V_{L}^{d}\right)_{43}^{\dagger}\left(V_{R}^{u}\right)_{33}+\kappa_{T}\left(V_{L}^{d}\right)_{43}^{\dagger}\left(V_{R}^{u}\right)_{53}+\kappa_{Q}\left(V_{L}^{d}\right)_{44}^{\dagger}\left(V_{R}^{u}\right)_{33}+\kappa\left(V_{L}^{d}\right)_{44}^{\dagger}\left(V_{R}^{u}\right)_{53}\right] \\
& -\sin \beta \cdot \bar{\lambda}\left(V_{L}^{d}\right)_{45}^{\dagger}\left(V_{R}^{u}\right)_{43}, \\
& \lambda_{t b_{4}}^{H^{ \pm}}=-\sin \beta \cdot\left[y_{b}\left(V_{L}^{u}\right)_{33}^{\dagger}\left(V_{R}^{d}\right)_{34}+\lambda_{B}\left(V_{L}^{u}\right)_{33}^{\dagger}\left(V_{R}^{d}\right)_{54}+\lambda_{Q}\left(V_{L}^{u}\right)_{34}^{\dagger}\left(V_{R}^{d}\right)_{34}+\lambda\left(V_{L}^{u}\right)_{34}^{\dagger}\left(V_{R}^{d}\right)_{54}\right] \\
& -\cos \beta \bar{\kappa}\left(V_{L}^{u}\right)_{35}^{\dagger}\left(V_{R}^{d}\right)_{44} \text {. } \\
& \Gamma\left(t_{i} \rightarrow H^{ \pm} b_{j}\right)=\frac{m_{t_{i}}}{32 \pi} \sqrt{\lambda\left(1, \frac{m_{H^{ \pm}}^{2}}{m_{t_{i}}^{2}}, \frac{m_{b_{j}}^{2}}{m_{t_{i}}^{2}}\right)}\left\{\left(\left|\lambda_{t_{i} b_{j}}^{H^{ \pm}}\right|^{2}+\left|\lambda_{b_{j} t_{i}}^{H^{ \pm}}\right|^{2}\right)\left(1+\frac{m_{b_{j}}^{2}-m_{H^{ \pm}}^{2}}{m_{t_{i}}^{2}}\right)\right. \\
& \left.+2\left(\left(\lambda_{t_{i} b_{j}}^{H^{ \pm}}\right)^{*} \lambda_{b_{j} t_{i}}^{H^{ \pm}}+\lambda_{t_{i} b_{j}}^{H^{ \pm}}\left(\lambda_{b_{j} t_{i}}^{H^{ \pm}}\right)^{*}\right) \frac{m_{b_{j}}}{m_{t_{i}}}\right\} \text {. }
\end{aligned}
$$

Open Access. This article is distributed under the terms of the Creative Commons Attribution License (CC-BY 4.0), which permits any use, distribution and reproduction in any medium, provided the original author(s) and source are credited. 